\title{
The acute time course of muscle and tendon tissue changes following one minute of static stretching
}

\author{
Andreas Konrad ${ }^{1} \&$ Markus Tilp ${ }^{1, *}$ \\ 1 Institute of Sport Science, University of Graz, Graz, Austria \\ * Corresponding author: Department of Sport Science, University of Graz, Mozartgasse 14/I, 8010 Graz, Austria \\ Tel: +43 (0) 316-3808332 \\ E-Mail: markus.tilp@uni-graz.at
}

\section{ORIGINAL ARTICLE}

\section{Article History:}

Submitted $16^{\text {th }}$ September 2019

Accepted $6^{\text {th }}$ March 2020

Published $19^{\text {th }}$ March 2020

Handling Editor:

Martin Kopp

University of Innsbruck, Austria

Editor-in-Chief:

Martin Kopp

University of Innsbruck, Austria

Reviewers:

Reviewer 1: anonymous

Reviewer 2: anonymous

\section{ABSTRACT}

The purpose of this study was to investigate the time course of the changes of various muscle and tendon mechanical properties and the function responses of the plantar flexor muscles following a single static stretching exercise for $1 \mathrm{~min}$.

Twenty-five healthy volunteers were assigned into a static stretching group or a control group. The static stretching group was tested with three different rest times ( $0 \mathrm{~min}, 20 \mathrm{~min}, 40 \mathrm{~min}$ ) after 2x30s of stretching. Controls were tested before and after a control period (10 min) without stretching. Dorsiflexion range of motion (RoM), passive resistive torque (PRT), and maximum voluntary contraction (MVC) were measured with a dynamometer. Ultrasonography of the medial gastrocnemius (GM) muscle-tendon junction (MTJ) displacement allowed to determine the length changes in the tendon and muscle, respectively, and hence to calculate their stiffness.

Following the stretching, we observed a significant increase in RoM directly following the stretching, 20 min post-stretching, and 40 min post-stretching. However, no changes were found in other functional parameters (PRT, MVC) or structural parameters (muscle and tendon stiffness). No changes were detected in any variable in the control group.

We conclude that a single static stretching exercise of $2 \times 30$ s increases the RoM for at least 40 min. However, this gain in RoM is not accompanied with more compliant muscle and/or tendon tissue, suggesting that $60 \mathrm{~s}$ of static stretching might not be stimulus enough to induce changes in the muscle-tendon structure. Hence, we speculate that other factors, such as increased stretch tolerance, might be responsible for the changes in the RoM observed in the present study.

Keywords:

stiffness - ultrasound - passive resistive torque - maximum voluntary contraction - range of motion - biomechanics

Citation:

Konrad, A., Tilp, M. (2020). The acute time course of muscle and tendon tissue changes following one minute of static stretching. Current Issues in Sport Science, 5:003. doi: 10.15203/CISS_2020.003.

\section{Introduction}

Static stretching exercises are commonly used in sports as a warm-up routine to increase the range of motion (RoM) of a joint (McHugh \& Cosgrave, 2010). Both the exercise intensity and especially the duration affect the outcome of the stretching. Besides a consistently observed increase in RoM, some studies have also reported a decrease in performance (e.g. peak isomeric torque), especially following stretching for durations longer than $1 \mathrm{~min}$ (Behm, Blazevich, Kay, \& McHugh, 2016; Kay \& Blazevich, 2012). The source of these effects seems to be on a muscular level rather than a tendon level. Kay, Husbands-Beasley and Blazevich (2015) reported decreased muscle stiffness (and no changes in tendon stiffness) following $4 \times 15$ s of static stretching, which could ex- 
plain the decrease in peak moment reported in Kay and Blazevich (2008). Decreased muscle stiffness following a single static stretching exercise has also been reported (Kay \& Blazevich, 2009; Konrad, Budini, \& Tilp, 2017a; Konrad, Stafilidis, \& Tilp, 2017b), but not by all authors (Kato, Kanehisa, Fukunaga, \& Kawakami, 2010; Kubo, Kanehisa, Kawakami, \& Fukunaga, 2001) who tried to find the mechanism behind the increase in RoM and a possible decrease in performance. Far fewer studies are available that analyzed the time course of the changes of different parameters of the muscletendon function and structure following the stretching.

Kay and Blazevich (2009) reported a decrease in muscle performance and PRT immediately after 3 min of stretching, which recovered $30 \mathrm{~min}$ after the stretching. These changes in the muscletendon function could be explained by changes of muscle stiffness immediately after the stretching, which also recovered $30 \mathrm{~min}$ after the stretching. Responses between immediately after and up to $30 \mathrm{~min}$ after the stretching were not investigated. We recently showed that 5 and 3 minutes of static stretching lead to significant decreases in muscle stiffness and PRT up to five minutes (Konrad, Reiner, Thaller, \&Tilp, 2019; Konrad \& Tilp, 2020) and tendencies for even longer effects following 5 minutes of stretching (Konrad et al., 2019). Although these stretching durations might apply for some types of sport like gymnastics, most other type of sports, e.g. team sports, apply much shorter durations for preparation which range from an average of $13.3 \mathrm{sec}$ in professional soccer players (Division 3) up to an average of $31.3 \mathrm{sec}$ (Premiere League) of static stretching in the hamstring muscles (Dabedo et al., 2004). Recently, Opplert, Genty and Babault (2016) also analyzed the time course of changes in several parameters following shorter stretching durations. They showed that static stretching of 1, 2, 3, 4, and $10 \times 30 \mathrm{~s}$ led to a decrease in PRT and muscle fascicle stiffness, immediately after and five min after the stretching. However, the authors did not measure beyond five minutes after stretching, leaving open how long such effects last. This is of specific interest as the time between stretching and competition exceeds five minutes in several types of sports (e.g. soccer).

Summarized, although there is already significant knowledge about the time course of structural effects of stretching with longer durations, it is still not clear how long such effects last for shorter durations and more sport practice relevant durations of stretching. Therefore, the objective of this study was to analyze the time course (immediately, $20 \mathrm{~min}$, and $40 \mathrm{~min}$ after stretching) of the acute changes in properties and function of the plantar flexor muscle-tendon system following a 1-min static stretching exercise. Based on the results presented we hypothesized an increase in RoM and a decrease in PRT immediately after and 20 min after stretching, but not 40 min after stretching. We further assumed that these functional changes in RoM and PRT would be accompanied by a decrease in muscle stiffness (Kay et al., 2015).

\section{Methods}

\section{Experimental design}

Participants visited the laboratory on two occasions. In the first session, the subjects were familiarized with the study and the methods used. During the second session, we assessed the effects of stretching immediately (0min_post), 20 min (20min_post), and 40 min (40min_post) after the stretching in the static stretching group. The control group performed no stretching and had to rest for $10 \mathrm{~min}$ in a sitting position. Before and after the static stretching or control conditions, the RoM, PRT, MVC torque, muscle-tendon stiffness, muscle stiffness, and passive and active tendon stiffness of the gastrocnemius medialis (GM) muscle were determined.

\section{Participants}

Fourteen healthy female (mean \pm SD; $24.1 \pm 2.7$ years, $166.7 \pm$ $4.8 \mathrm{~cm}, 58.6 \pm 6.4 \mathrm{~kg}$ ) and eleven healthy male (mean $\pm \mathrm{SD}$; $25.9 \pm 6.9$ years, $181.2 \pm 6.4 \mathrm{~cm}, 75.2 \pm 6.7 \mathrm{~kg}$ ) volunteers with no history of lower leg injuries participated in the study. All subjects were athletes from different kind of sports and performed on average $6.1 \pm 2.2$ hours sports per week. Subjects were assigned either into a static stretching group ( $\mathrm{n}=11 ; 7$ females/ 4 males) or a control group ( $n=14 ; 7$ females/ 7 males). Subjects were informed about the testing procedure, but were naive of the study's aim and hypotheses.

The study was approved by the local research ethics board, and written informed consent was obtained from all volunteers before the onset of the experimental procedures.

\section{Measures}

The temperature in the laboratory was kept constant at around $20.5^{\circ} \mathrm{C}$. Measurements were performed without any warm-up and in the following order: pre-tests: RoM (1-min rest), PRT (1-min rest), MVC (1-min rest); intervention: stretching for $2 \times 30 \mathrm{~s}$; post-test: immediately following the stretching and after 20 min of rest and 40 min of rest in the same order (RoM (1-min rest), PRT (1-min rest), $\mathrm{MVC})$. For the control group, the post-tests were performed without prior stretching, $10 \mathrm{~min}$ after the pre-tests.

Range of motion (RoM) measurement. RoM was determined with an isokinetic dynamometer (CON-TREX MJ, CMV AG, Duebendorf, Switzerland), with the standard setup for ankle joint movement individually adjusted. Subjects were seated with a hip joint angle of $110^{\circ}$, with the foot resting on the dynamometer foot plate (barefooted) and the knee fully extended. Two oblique straps on the upper body and one strap around the thigh were used to secure the participant to the dynamometer and exclude any evasive movement. The foot was fixed with a strap to the dynamometer foot plate, and the estimated ankle joint center was carefully aligned with the axis of the dynamometer to avoid any heel displacement. Participants were moved to the neutral ankle joint position in the 
dynamometer ( $90^{\circ}$ between foot sole and tibia) and were subsequently asked to regulate the motor of the dynamometer with a remote control to get into a dorsiflexion (stretching) position at $5 \% \mathrm{~s}$ until the point of discomfort was reached. The difference between neutral position and the maximum dorsiflexion was defined as the dorsiflexion RoM.

Passive resistive torque (PRT) measurement. During this measurement, the dynamometer moved the ankle joint from a $20^{\circ}$ plantar flexion to the individual maximum dorsiflexion RoM which was previously determined in the RoM measurement. During pilot measurements, we recognized a conditioning effect during the first two passive movements, similar to the active conditioning reported by Maganaris (2003). Therefore, the ankle joint was moved passively for three cycles and measurements were taken during the third cycle, to minimize bias due to conditioning effects. Similar to the studies by Kubo, Kanehisa, \& Fukunaga (2002) and Mahieu, Cools, De Wilde, Boon and Witvrouw (2009), the velocity of the dynamometer was set to $5 \%$ to exclude any reflexive muscle activity. Participants were asked to relax during the measurements.

Maximum voluntary contraction (MVC) measurement. MVC measurement was performed with the dynamometer at an ankle position of $10^{\circ}$ of plantar flexion. Participants were instructed to perform two isometric MVCs of the plantar flexors for $5 \mathrm{~s}$, with rest periods of at least $1 \mathrm{~min}$ between the measurements to avoid any fatigue. The attempt with the highest MVC torque value was taken for further analysis.

Electromyography (EMG). Muscular activity was monitored by EMG (myon 320, myon AG, Zurich, Switzerland) during PRT and MVC measurements. After standard skin preparation, surface electrodes (Blue Sensor N, Ambu A/S, Ballerup, Denmark) were placed on the muscle bellies of the GM and the tibialis anterior, according to SENIAM recommendations (Hermens et al., 1999.). In the PRT measurements, the raw EMG was monitored online to ensure that the subject was relaxed.

Measurement of elongation of the muscle-tendon structures. A real-time ultrasound apparatus (mylab 60, Esaote S.p.A., Genova, Italy) with a 10-cm B-mode linear-array probe (LA 923, Esaote S.p.A., Genova, Italy) was used to obtain longitudinal ultrasound images of the GM.

During the PRT and MVC measurements, the ultrasound probe was placed on the distal end of the GM (as described in a previous study, Konrad, Gad, \& Tilp, 2014a, see Fig. 2), where the muscle merges into the Achilles tendon, i.e. the muscle-tendon junction (MTJ) (Kato et al., 2010). The ultrasound probe was attached to the lower leg with a custom-built styrofoam block and secured with elastic bands to prevent any displacement of the probe. The previous studies (Konrad et al., 2017a; Stafilidis and Tilp, 2015), confirmed that this kind of fixation of the ultrasound probe did not lead to any unwanted shifts of the probe during the measurement. To determine the muscle displacement during PRT measurement, the echoes of the MTJ in the ultrasound videos were manually tracked (Kato et al., 2010). Similar to the approach used by other authors (Kato et al., 2010; Morse, Degens, Seynnes, Maganaris, \& Jones, 2008), the cadaveric regression model of Grieve, Gavanagh and Pheasant (1978) was used to obtain the length changes of the muscle-tendon unit (MTU) of the GM during passive movements. The difference between the overall MTU length change and the displacement of the muscle was defined as the tendon displacement. To determine the tendon displacement during MVC measurement, the echoes of a fascicle insertion at the deep aponeurosis near the MTJ were manually tracked (Konrad \& Tilp, 2014b; Kubo et al., 2002). This was necessary because the bulging of the muscle during the contraction led to poor video quality and restricted the exact determination of the MTJ in some individuals. The ultrasound images were recorded at $25 \mathrm{~Hz}$. During PRT and MVC measurements, the videos were synchronized with the other data with a custom-built manual trigger. The videos were cut and digitized in VirtualDub open-source software (version 1.6.19, www.virtual dub.org) and analyzed in ImageJ open-source software (version 1.44p, National Institutes of Health, U.S.). Each video was analyzed by two investigators, and the mean values of the measurements were used for further analysis of the muscle-tendon structure. The mean ICCs of the inter-rater test of the ultrasound video analysis were 0.98 and 0.97 for the MTJ displacement during PRT and MVC measurements, respectively. Except for the principal investigator, the investigators were neither informed of the hypotheses of the study nor the group allocation of the subjects. During the analysis of the PRT measurement, every fifth frame, and for MVC measurement every second frame, were analyzed by the investigators, corresponding to a time resolution of 0.2 and $0.08 \mathrm{~s}$, respectively.

Calculation of muscle/tendon force, passive muscle/tendon stiffness, active tendon stiffness, and muscle-tendon stiffness. The muscle force of the GM was estimated by multiplying the measured torque by the relative contribution of the physiological crosssectional area (18\%) of the GM within the plantar flexor muscles (Kubo et al., 2002; Mahieu et al., 2009), and dividing by the moment arm of the triceps surae muscle, which was individually measured by tape measure as the distance between the malleolus lateralis and the Achilles tendon at rest at neutral ankle position $\left(90^{\circ}\right)$ (Konrad \& Tilp, 2014b).

Active tendon stiffness was calculated as the change in the active force divided by the change of the related tendon length during the MVC measurements over a range of force of $50-90 \%$ of MVC (Kay et al., 2015) at $10^{\circ}$ plantar flexion. Passive tendon stiffness, muscle stiffness, and muscle-tendon stiffness were calculated as the change in passive force produced at the last $10^{\circ}$ up to maximum dorsiflexion divided by the change of the related tendon length, muscle length, and joint angle, respectively. In accordance with Magnusson, Simonsen, Aagard, Boesen, Sørensen and Kjaer (1997), the stretching maximum of the pre-test was also taken in the post-test to allow a comparison. 


\section{Stretching exercise}

The stretching exercise was undertaken with the dynamometer, with the starting point at neutral ankle position $\left(90^{\circ}\right)$. The subjects were asked to regulate the motor of the dynamometer with a remote control to get into a dorsiflexion (stretching) position corresponding to the maximum RoM, with the help of visual feedback. This position was held for $30 \mathrm{~s}$. This procedure was then repeated, resulting in a total stretch period of $60 \mathrm{~s}$. Between the stretches, the dynamometer moved the ankle into neutral position and back again into the stretching position at $5 \%$ s. Hence, the break between the stretches lasted around $20 \mathrm{~s}$.

\section{Statistical analyses}

SPSS (version 20.0, SPSS Inc., Chicago, Illinois) was used for all the statistical analyses. To determine the inter-rater reliability of the muscle-tendon displacement measurements, intraclass correlation coefficients (ICCS) were used. A Shapiro-Wilk test was used to verify the normal distribution of all the variables. To confirm homogeneity of the baseline characteristics between the exercise and the control groups, a t-test was performed. The data of the control group before and after the control period were compared by paired t-tests. For the static stretching group, we performed a oneway repeated measures ANOVA to test for differences between the time points. If the ANOVA test was significant, we performed a t-test (Bonferroni corrected) to identify the location of the significant differences. If the data were not normally distributed, the respective non-parametric tests were applied (Friedmann test, Mann-Whitney U-test, Wilcoxon test). An alpha level of $\mathrm{P}=0.05$ was defined for the statistical significance of all the tests.

\section{Results}

\section{Range of motion (RoM)}

The repeated measures ANOVA indicated significant differences in the RoM between the time points $\left(P<0.01, F=11.67, \eta^{2}=0.81\right)$. The post-hoc analysis revealed differences between the baseline RoM and values at 0min_post $(+12.4 \%$; $\mathrm{P}<0.01)$, 20min_post $(+11.1 \%$; $P<0.01)$, and 40min_post $(+13.0 \% ; P<0.01)$ (see Table $1)$. No changes were detected in the control group (see Table 2 ).

Passive resistive torque (PRT) and the related muscle-tendon structure parameters

No changes were detected in PRT, muscle-tendon stiffness, muscle stiffness, and passive tendon stiffness in the static stretching group (see Table 1). Moreover, no changes were detected in the control group (see Table 2).

Table 1. Results of maximum dorsiflexion RoM, as well as PRT, passive tendon stiffness, muscle stiffness, muscle-tendon stiffness, MVC torque, and active tendon stiffness of the static stretching group at all time points.

\begin{tabular}{|c|c|c|c|c|c|c|c|c|c|}
\hline \multirow{2}{*}{$\begin{array}{l}\text { Static stretching group } \\
\text { Range of motion }\left(^{\circ}\right)\end{array}$} & \multicolumn{2}{|c|}{ PRE } & \multicolumn{2}{|c|}{ Omin_post } & \multicolumn{2}{|c|}{ 20min_post } & \multicolumn{2}{|c|}{ 40min_post } & \multirow{2}{*}{$\frac{P}{0.00 \#}$} \\
\hline & 35.0 & $(5.0)$ & 39.3 & $(6.8)^{*}$ & 38.8 & $(6.2)^{*}$ & 39.5 & $5.1)^{*}$ & \\
\hline Passive resistive torque ( $\mathrm{Nm}$ ) & 29.6 & $(14.0)$ & 29.0 & $(15.0)$ & 28.4 & $(15.1)$ & 28.7 & 14.1) & 0.08 \\
\hline Passive tendon stiffness $(\mathrm{N} / \mathrm{mm})$ & 13.6 & (6.9) & 14.5 & $(7.9)$ & 14.4 & (9.4) & 13.7 & $(6.5)$ & 0.99 \\
\hline Muscle stiffness (N/mm) & 9.7 & (3.9) & 9.1 & $(4.2)$ & 9.7 & (3.8) & 9.5 & $(4.0)$ & 0.32 \\
\hline Muscle-tendon stiffness $\left(\mathrm{Nm} /{ }^{\circ}\right)$ & 1.5 & $(0.8)$ & 1.5 & $(0.9)$ & 1.4 & $(0.8)$ & 1.5 & $(0.8)$ & 0.09 \\
\hline MVC torque (Nm) & 86.5 & $(33.3)$ & 81.0 & $(27.3)$ & 81.1 & $(30.2)$ & 80.0 & $(25.0)$ & 0.24 \\
\hline Active tendon stiffness $(\mathrm{N} / \mathrm{mm})$ & 26.6 & $(13.4)$ & 26.2 & $(15.7)$ & 25.8 & $(10.0)$ & 25.8 & $(14.0)$ & 0.71 \\
\hline
\end{tabular}

\#= significant difference in analysis of variance, ${ }^{*}=$ significant difference to pre-value data, mean \pm SD.

Table 2. Results of maximum dorsiflexion RoM, as well as PRT, passive tendon stiffness, muscle stiffness, muscle-tendon stiffness, MVC torque, and active tendon stiffness of the control group. Post-assessment of the control group was performed after a 10 min break between the last measurement in the pre-assessment and the beginning of the post-assessment, mean \pm SD.

\begin{tabular}{lccccc}
\hline Control group & \multicolumn{3}{c}{ PRE } & \multicolumn{3}{c}{ POST } & P \\
\hline Range of motion $\left({ }^{\circ}\right)$ & 30.7 & $(6.7)$ & 30.1 & $(6.9)$ & 0.21 \\
Passive resistive torque $(\mathrm{Nm})$ & 27.7 & $(9.3)$ & 27.1 & $(10.2)$ & 0.75 \\
Passive tendon stiffness $(\mathrm{N} / \mathrm{mm})$ & 17.8 & $(5.7)$ & 18.8 & $(10.4)$ & 0.79 \\
Muscle stiffness $(\mathrm{N} / \mathrm{mm})$ & 7.9 & $(2.4)$ & 7.7 & $(2.0)$ & 0.69 \\
Muscle-tendon stiffness $\left(\mathrm{Nm} /{ }^{\circ}\right)$ & 1.5 & $(0.6)$ & 1.4 & $(0.6)$ & 0.72 \\
MVC torque $(\mathrm{Nm})$ & 90.8 & $(27.8)$ & 92.5 & $(28.8)$ & 0.51 \\
Active tendon stiffness $(\mathrm{N} / \mathrm{mm})$ & 28.8 & $(10.3)$ & 28.9 & $(14.2)$ & 0.98 \\
\hline
\end{tabular}




\section{Maximum voluntary contraction (MVC) and active tendon stiffness}

No changes were detected in MVC and active tendon stiffness in the static stretching group (see Table 1). Moreover, no changes were detected in the control group (see Table 2).

\section{Discussion}

Previous studies showed the time course of effects following longer stretching durations ( $>2 \mathrm{~min}$ ) and the short time effect (up to $5 \mathrm{~min}$ following stretching) following short stretching durations ( $<=1 \mathrm{~min}$ ). Therefore, the purpose of this study was to investigate the time course (immediately after stretching $=0$ min_post, 20 min after stretching $=20 \mathrm{~min} \_$post, and $40 \mathrm{~min}$ after stretching $=$ 40 min_post) of possible acute changes of the MTU function and the structure of the plantar flexor muscles following a 1-min static stretching exercise to get a full picture of the dose-response relationship of stretching. We observed an increased RoM over the whole time course (0min_post, 20min_post, 40min_post); however, against our hypothesis, this could not be explained by changes in the muscle-tendon tissue properties. Moreover, no changes in functional parameters (PRT or MVC) could be detected at any time point after the stretching exercise.

In the present study, similar to previous studies of a single static stretching exercise (Kato et al., 2010; Kay \& Blazevich, 2008; Konrad et al., 2017a; Konrad et al., 2017b), RoM was found to be increased immediately after the stretching. The increase in RoM after 20 min and 40 min breaks following the stretching is in accordance with the study of Mizuno, Matsumoto and Umemura (2013), who reported that the retention time of the RoM following a 5-min stretching exercise is between 30 and $60 \mathrm{~min}$. Bringing both studies together, i.e. the findings of Mizuno et al. (2013) and the findings of the present study, it seems that the increase in RoM following a single static stretching exercise lasts between 40 and $60 \mathrm{~min}$. However, in contrast, Ryan et al. (2008) reported that RoM returned to baseline values after $10 \mathrm{~min}$, following 2, 4, and $8 \mathrm{~min}$ of stretching. Although Ryan et al. (2008) performed the passive stretching in a similar way to the present study, subjects showed an increase in RoM of about $7.7 \%$ following $4 \times 30$ s of stretching compared to $12.3 \%$ following $2 \times 30 \mathrm{~s}$ in the present study. This might indicate that the intensity of the stretching exercise, i.e. the point of discomfort for the individuals, was lower compared to the present study. However, when the individuals in the study of Ryan et al. (2008) stretched for $8 \times 30 \mathrm{~s}$ and $16 \times 30 \mathrm{~s}$, they increased their RoM by a similar percentage ( $13.9 \%$ and $13.5 \%$, respectively), but RoM also returned to baseline values $10 \mathrm{~min}$ after the stretching. A possible explanation for this could be the electrical stimulations applied during their experiments. Supramaximal doublets were administered during the MVC plateau and again 3-5 s after the MVC trial at rest. These stimulations might have decreased the pain threshold for the individuals, who might not have been familiar with electrical stimulation for all conditions, which might have affected the results.
While some authors (Herda, Cramer, Ryan, McHugh, \& Stout, 2008; Marek et al., 2005; Konrad et al., 2019) have reported a decrease in maximum strength performance following a single static stretching exercise, the results of others and the current study suggest that the maximum performance is not negatively affected (Konrad et al., 2017a; Konrad \& Tilp, 2020; Kubo et al., 2001; Zakas, Doganis, Papakonstandinou, Sentelidis, \& Vamvakoudis, 2006). These controversial results could possibly be explained by the differences in overall stretch duration, as summarized in the reviews by Kay and Blazevich (2012) and Behm et al. (2016), who pointed out that only stretching for $60 \mathrm{~s}$ or longer can induce a detrimental effect on maximum performance. Our observation that maximum strength performance (MVC) was not decreased up to $40 \mathrm{~min}$ also excludes a possible delayed effect of $60 \mathrm{~s}$ of stretching.

PRT was not changed at any time point after the single static stretching exercise. This is in accordance with some other studies (Magnusson, Aagard, Simonsen, \& Bojsen-Møller 1998), but not all the previous studies dealing with similar (Kay et al., 2015; Opplert et al., 2016) or higher durations of stretching (Kay \& Blazevich, 2009; Konrad et al., 2017a; 2017b; 2019; Konrad \& Tilp, 2020; Nakamura, Ikezoe, Takeno, \& Ichihashi, 2013; Opplert et al., 2016). The controversial results of Kay et al. (2015), who also considered 1 min of total stretching, might be explained by the different stretching approaches. While Kay et al. (2015) adapted the joint angle after every $15 \mathrm{~s}$ bout of stretching, in the current study, stretching with constant joint angle was performed. In a previous study, we demonstrated that the adapted-angle stretching applied by Kay et al. (2015) is a more efficient way to decrease PRT (and increase RoM) than the constant-angle stretching applied in the present study (Konrad et al., 2017a). Additionally, we have to mention that the level of significance for PRT indicates a tendency toward a decrease $(P=0.08$, see Table 1$)$. Therefore, one could assume that a more intensive stretching exercise (stretching with adapted angle or constant torque) or an increase in stretching duration might have led to a significant decrease in PRT.

PRT ranges (14.2 - 56.3, 11.8 - 54.0, 12.1 - 54.4, 11.1 - $50.5 \mathrm{Nm}$ for pre/0/20/40 min post, respectively) and muscle-tendon stiffness ranges $\left(0.74-2.79,0.61-2.98,0.55-2.74,0.59-2.68 \mathrm{Nm} /{ }^{\circ}\right.$ for pre/0/20/40 min post, respectively) stayed essentially constant following the stretching. A muscle-tendon stiffness of $1.5 \mathrm{Nm} /{ }^{\circ}$ means that the last $10^{\circ}$ of passive dorsiflexion was accompanied by a change of $15 \mathrm{Nm}$. A further increase of $4^{\circ}$ of plantar flexion, as approximately observed in the present study, only explained by a change in muscle-tendon stiffness would have required a stiffness value of approx. $1.07 \mathrm{Nm} /{ }^{\circ}\left(=15 \mathrm{Nm} / 14^{\circ}\right)$. Similarly, the observed muscle and tendon stiffness differences are far too small to explain the changes in RoM.

This was against our hypothesis and the findings of several studies that have reported changes in the muscle (Kay \& Blazevich, 2009; Kay et al., 2015; Konrad et al., 2017a; 2017b; 2019; Konrad \& Tilp, 2020; Opplert et al., 2016) or tendon structure (Kato et al., 2010; Kubo et al., 2001) due to stretching. However, other studies (Nakamura et al., 2013; Stafilidis and Tilp, 2015) are in accordance with our findings and reported no changes in the muscular or tendinous tissue following stretching of up to $60 \mathrm{~s}$. Nakamura et al. 
(2013) recommended in their study that $120 \mathrm{~s}$ of static stretching is required to change muscle extensibility. Summarizing, it appears probable that only longer durations of a single static stretching exercise, as performed by Kay and Blazevich (2009; $3 \times 60$ s), Konrad et al. (2017a; $4 \times 30$ s), Konrad et al. (2017b; $4 \times 30$ s), Konrad et al., (2019; 5 x 60 s), Konrad and Tilp (2020; $3 \times 60$ s), Kato et al. (2010; $20 \mathrm{~min})$, Kubo et al. (2001;10 min), and Nakamura et al. (2013; 2 min to $5 \mathrm{~min}$ ), lead to structural adaptations of the MTU, while shorter durations, as performed by Stafilidis and Tilp (2015; $15 \mathrm{~s}$ or $60 \mathrm{~s})$, Nakamura et al. (2013; $60 \mathrm{~s})$, and the present study $(2 \times$ $30 \mathrm{sec})$, have no detectable effect on the muscle and tendon structure. A possible explanation why Kay et al. (2015) found decreased muscle stiffness following $4 \times 15 \mathrm{~s}$ of static stretching might be the more intensive stretching approach used compared to the present study. Nevertheless, it is still not clear which mechanism can explain the increase in RoM following a single static stretching exercise of $1 \mathrm{~min}$ over the time course of at least $40 \mathrm{~min}$. According to our results, we can assume that increased stretch tolerance (Magnusson, Simonsen, Aagard, Sørensen, \& Kjaer, 1996) might be a possible explanation for the gain in flexibility (RoM).

However, independent of the mechanism responsible for the increase in RoM, the results of this study provide evidence that an acute bout of static stretching for $2 \times 30 \mathrm{~s}$ can induce an increase in RoM (beneficial for many kind of sports) without a detrimental effect on the MTU (i.e. decrease in MVC) up to 40 minutes after the stretching. Therefore, according to our results, athletes with a high demand on joint flexibility (e.g. in gymnastics) and strength should stretch up to $60 \mathrm{~s}$ to increase the RoM of their joints for a prolonged effect for at least 40 minutes. Since the likelihood of a detrimental effect increases with stretch duration, we would not recommend to stretch more than $60 \mathrm{~s}$ prior to a training or competition as also pointed out in some recent reviews (Kay \& Blazevich, 2012; Behm et al., 2016).

We conclude that a single static stretching exercise performed over $1 \mathrm{~min}$ increases the RoM for at least $40 \mathrm{~min}$, without loss of muscle performance, during isometric contractions. However, these changes cannot be explained by more compliant muscle and/or tendon tissue. Hence, the increased RoM observed in the present study might be associated with increased stretch tolerance (Magnusson et al., 1996).

\section{Funding}

This study was supported by a grant (Project $P$ 27665) from the Austrian Science Fund (FWF).

\section{Competing Interests}

The authors have declared that no competing interests exist.

\section{Data Availability Statement}

All relevant data are within the paper.

\section{References}

Behm, D. G., Blazevich, A. J., Kay, A. D., \& McHugh, M. (2016). Acute effects of muscle stretching on physical performance, range of motion, and injury incidence in healthy active individuals: a systematic review. Applied physiology, nutrition, and metabolism, 41(1), 1-11.

Grieve, D., Gavanagh, P., \& Pheasant, S. (1978). Prediction of gastrocnemius length from knee and ankle joint posture. In: Asmussen E., Jorgensen K., Eds. Biomechanics, VI-A. Baltimore: University Park Press, 405-412.

Herda, T. J., Cramer, J. T., Ryan, E. D., McHugh, M. P., \& Stout, J. R. (2008). Acute Effects of Static versus Dynamic Stretching on Isometric Peak Torque, Electromyography, and Mechanomyography of the Biceps Femoris Muscle. Journal of Strength and Conditioning Research, 22(3), 809-817. doi: 10.1519/ JSC.0b013e31816a82ec

Hermens, H. J., Freriks, B., Merletti, R., Stegeman, D., Blok, J., Rau, G., ... Hägg, G. (n.d.). European Recommendations for Surface ElectroMyoGraphy Results of the SENIAM project. Retrieved from http://www.seniam.org/pdf/contents8.PDF

Kato, E., Kanehisa, H., Fukunaga, T., \& Kawakami, Y. (2010). Changes in ankle joint stiffness due to stretching:The role of tendon elongation of the gastrocnemius muscle. European Journal of Sport Science, 10(2), 111-119. doi: 10.1080/17461390903307834

Kay, A. D., \& Blazevich, A. J. (2008). Reductions in active plantarflexor moment are significantly correlated with static stretch duration. European Journal of Sport Science, 8(1), 41-46. doi: 10.1080/17461390701855505

Kay, A. D., \& Blazevich, A. J. (2009). Moderate-duration static stretch reduces active and passive plantar flexor moment but not Achilles tendon stiffness or active muscle length. Journal of Applied Physiology (Bethesda, Md. : 1985), 106(4), 1249-1256. doi: 10.1152/japplphysiol.91476.2008

Kay, A. D., \& Blazevich, A. J. (2012). Effect of Acute Static Stretch on Maximal Muscle Performance: A Systematic Review. Medicine and Science in Sports and Exercise, 44(1), 154-164. doi: 10.1249/ MSS.0b013e318225cb27

Kay, A. D., Husbands-Beasley, J., \& Blazevich, A. J. (2015). Effects of Contract-Relax, Static Stretching, and Isometric Contractions on Muscle-Tendon Mechanics. Medicine and Science in Sports and Exercise, 47(10), 2181-2190. doi: 10.1249/ MSS.0000000000000632

Konrad, A., Gad, M., \& Tilp, M. (2014a). Effect of PNF stretching training on the properties of human muscle and tendon structures. Scandinavian Journal of Medicine \& Science in Sports, 1-10. doi: $10.1111 / \mathrm{sms} .12228$

Konrad, A., \& Tilp, M. (2014b). Effects of ballistic stretching training on the properties of human muscle and tendon structures. 
Journal of Applied Physiology (Bethesda, Md. : 1985), (May), 2935. doi: 10.1152/japplphysiol.00195.2014

Konrad, A., Budini, F., \& Tilp, M. (2017a). Acute effects of constant torque and constant angle stretching on the muscle and tendon tissue properties. European Journal of Applied Physiology, 117(8), 1649-1656. doi: 10.1007/s00421-017-3654-5

Konrad, A., Stafilidis, S., \& Tilp, M. (2017b). Effects of acute static, ballistic, and PNF stretching exercise on the muscle and tendon tissue properties. Scandinavian Journal of Medicine and Science in Sports, 27(10), 1070-1080. doi: 10.1111/sms.12725

Konrad, A., Reiner, M. M., Thaller, S., \& Tilp, M. (2019). The time course of muscle-tendon properties and function responses of a five-minute static stretching exercise. European journal of sport science, 19(9) 1195-1203.

Konrad, A., \& Tilp, M. (2020). The time course of muscle-tendon unit function and structure following three minutes of static stretching. Journal of Sports Science \& Medicine (19), 52-58.

Kubo, K., Kanehisa, H., Kawakami, Y., \& Fukunaga, T. (2001). Influence of static stretching on viscoelastic properties of human tendon structures in vivo Influence of static stretching on viscoelastic properties of human tendon structures in vivo. Journal of Applied Physiology, 90, 520-527. Retrieved from http:// www.ncbi.nlm.nih.gov/pubmed/11160050

Kubo, K., Kanehisa, H., \& Fukunaga, T. (2002). Effect of stretching training on the viscoelastic properties of human tendon structures in vivo. Journal of Applied Physiology (Bethesda, Md. : 1985), 92(2), 595-601. doi: 10.1152/japplphysiol.00658.2001

Maganaris, C. N. (2003). Tendon conditioning: artefact or property? Proceedings. Biological Sciences / The Royal Society, 270 Suppl, S39-S42. doi: 10.1098/rsbl.2003.0004

Magnusson, S. P., Simonsen, E. B., Aagaard, P., Sørensen, H., \& Kjaer, M. (1996). A mechanism for altered flexibility in human skeletal muscle. The Journal of Physiology, 497 (Pt 1(1996), 291-298.

Magnusson, S. P., Simonsen, E. B., Aagaard, P., Boesen, J., Johannsen, F., \& Kjaer, M. (1997). Determinants of musculoskeletal flexibility: viscoelastic properties, cross-sectional area, EMG and stretch tolerance. Scandinavian Journal of Medicine \& Science in Sports, 7(4), 195-202.

Magnusson, S. P., Aagard, P., Simonsen, E., \& Bojsen-Møller, F. (1998). A biomechanical evaluation of cyclic and static stretch in human skeletal muscle. International Journal of Sports Medicine, 19(5), 310-316. doi: 10.1055/s-2007-971923

Mahieu, N. N., Cools, a., De Wilde, B., Boon, M., \&Witvrouw, E. (2009). Effect of proprioceptive neuromuscular facilitation stretching on the plantar flexor muscle-tendon tissue properties. Scandinavian Journal of Medicine and Science in Sports, 19(4), 553-560. doi: 10.1111/j.1600-0838.2008.00815.x

Marek, S. M., Cramer, J. T., Fincher, A. L., Massey, L. L., Dangelmaier, S. M., Purkayastha, S., ... Culbertson, J. Y. (2005). Acute Effects of Static and Proprioceptive Neuromuscular Facilitation Stretching on Muscle Strength and Power Output. Journal of Athletic Training, 40(2), 94-103. Retrieved from http://www.ncbi.nlm. nih.gov/pubmed/15970955

McHugh, M. P., \& Cosgrave, C. H. (2010). To stretch or not to stretch: The role of stretching in injury prevention and performance.
Scandinavian Journal of Medicine and Science in Sports, 20(2), 169-181. doi: 10.1111/j.1600-0838.2009.01058.x

Mizuno, T., Matsumoto, M., \& Umemura, Y. (2013). Viscoelasticity of the muscle-tendon unit is returned more rapidly than range of motion after stretching. Scandinavian Journal of Medicine \& Science in Sports, 23(1), 23-30. doi: 10.1111/j.16000838.2011.01329.x

Morse, C. I., Degens, H., Seynnes, O. R., Maganaris, C. N., \& Jones, D. a. (2008). The acute effect of stretching on the passive stiffness of the human gastrocnemius muscle tendon unit. The Journal of Physiology, 586(1), 97-106. doi: 10.1113/jphysiol.2007.140434

Nakamura, M., Ikezoe, T., Takeno, Y., \& Ichihashi, N. (2013). Time course of changes in passive properties of the gastrocnemius muscle-tendon unit during $5 \mathrm{~min}$ of static stretching. Manual Therapy, 18(3), 211-215. doi: 10.1016/J.MATH.2012.09.010

Opplert, J., Genty, J.-B., \& Babault, N. (2016). Do Stretch Durations Affect Muscle Mechanical and Neurophysiological Properties? International Journal of Sports Medicine, 37(9), 673-679. doi: 10.1055/s-0042-104934

Ryan, E. D., Beck, T. W., Herda, T. J., Hull, H. R., Hartman, M. J., Stout, J. R., \& Cramer, J. T. (2008). Do practical durations of stretching alter muscle strength? A dose-response study. Medicine and Science in Sports and Exercise, 40(8), 1529-1537. doi: 10.1249/ MSS.0b013e31817242eb

Stafilidis, S., \&Tilp, M. (2015). Effects of short duration static stretching on jump performance, maximum voluntary contraction, and various mechanical and morphological parameters of the muscle-tendon unit of the lower extremities. European Journal of Applied Physiology, 115(3), 607-617. doi: 10.1007/s00421014-3047-y

Zakas, A., Doganis, G., Papakonstandinou, V., Sentelidis, T., \& Vamvakoudis, E. (2006). Acute effects of static stretching duration on isokinetic peak torque production of soccer players. Journal of Bodywork and Movement Therapies, 10(2), 89-95. doi: 10.1016/J.JBMT.2005.04.007 
